\title{
Retraction Note to: Writer identification using graphemes
}

\author{
MANOJ KUMAR SHARMA* and VANSHIKA CHANDERIYA \\ Department of Computer and Communication Engineering, School of Computer Science and Information \\ Technology, Manipal University Jaipur, Dehmi Kalan, Off Jaipur-Ajmer Expressway, Jaipur 303007, India. \\ *Corresponding author. E-mail: manojkumar.sharma@jaipur.manipal.edu; \\ vanshi2000chanderiya@gmail.com
}

Published online 4 November 2020

Retraction Note to: Sādhanā (2020) 45:42

https://doi.org/10.1007/s12046-020-1276-9

The Editor-in-Chief has retracted this article [1] because it contains material that substantially overlaps with another publication [2].

The author (M K Sharma) does not agree to this retraction. The author (V Chanderiya) has not responded.

\section{References}

[1] Sharma M K, Chanderiya V 2020 Writer identification using graphemes. Sädhanā 45:42. https://doi.org/10.1007/s12046020-1276-9

[2] Aubin V, Mora M, Santos-Peñas M 2018 Off-line writer verification based on simple graphemes. Pattern Recognit. 79:414-426. https://doi.org/10.1016/j.patcog.2018.02.024

The original article can be found online at https:// doi.org/10.1007/s12046-020-1276-9.

*For correspondence 